\title{
Anisotropic pH-Responsive Hydrogels Containing Soft or Hard Rod- Like Particles Assembled Using Low Shear
}

Amir H. Milani, ${ }^{*},{ }^{\dagger}$ Lee A. Fielding, ${ }^{\dagger}$ Polly Greensmith, ${ }^{\dagger}$ Brian R. Saunders, ${ }^{*}{ }^{\dagger} \odot$ Daman J. Adlam, ${ }^{*}$ Anthony J. Freemont, ${ }^{\ddagger}, \S$ Judith A. Hoyland, ${ }^{\ddagger, \S}$ Nigel W. Hodson, ${ }^{\|}$Mohamed A. Elsawy, ${ }^{\dagger, \perp}$ Aline F. Miller, ${ }^{\perp, \#}$ Liam P. D. Ratcliffe, ${ }^{\nabla}$ Oleksandr O. Mykhaylyk, ${ }^{\nabla}$ and Steven P. Armes ${ }^{\nabla}(\mathbb{0}$

${ }^{\dagger}$ School of Materials, University of Manchester, Manchester, M13 9PL, United Kingdom

${ }^{\ddagger}$ Division of Cell Matrix Biology and Regenerative Medicine, Faculty of Biology, Medicine and Health, University of Manchester, Oxford Road, Manchester, M13 9PT, United Kingdom

${ }^{\S}$ NIHR Manchester Musculoskeletal Biomedical Research Unit, Manchester Academic Health Science Centre, Manchester, United Kingdom

"BioAFM Facility, Faculty of Biology, Medicine and Health, Stopford Building, University of Manchester, Oxford Road, Manchester, M13 9PT, United Kingdom

${ }^{\perp}$ Manchester Institute of Biotechnology, University of Manchester, Manchester, M13 9PL, United Kingdom

${ }^{\#}$ School of Chemical Engineering and Analytical Science, University of Manchester, Manchester, M13 9PL, United Kingdom

${ }^{\nabla}$ Department of Chemistry, The University of Sheffield, Dainton Building, Brook Hill, Sheffield, South Yorkshire S3 7HF, United Kingdom

\section{Supporting Information}

ABSTRACT: A simple and versatile low-shear approach for assembling hydrogels containing aligned rod-like particles (RLPs) that are birefringent and exhibit $\mathrm{pH}$-triggered anisotropic swelling is developed. Anisotropic composite hydrogels are prepared by applying low shear $\left(0.1 \mathrm{~s}^{-1}\right)$ to mixtures of $\mathrm{pH}$-responsive nanogels (NGs) and RLPs. The NGs, which contained high methacrylic acid contents, acted as both shear transfer vehicles and macro-cross-linkers for anisotropic gel formation. Three model RLP systems are investigated: (i) soft triblock copolymer worms, (ii) stiff selfassembled $\beta$-sheet peptide fibers, and (iii) ultrahigh modulus nanocrystalline cellulose fibers. RLP alignment was confirmed using polarized light imaging, atomic force microscopy, and

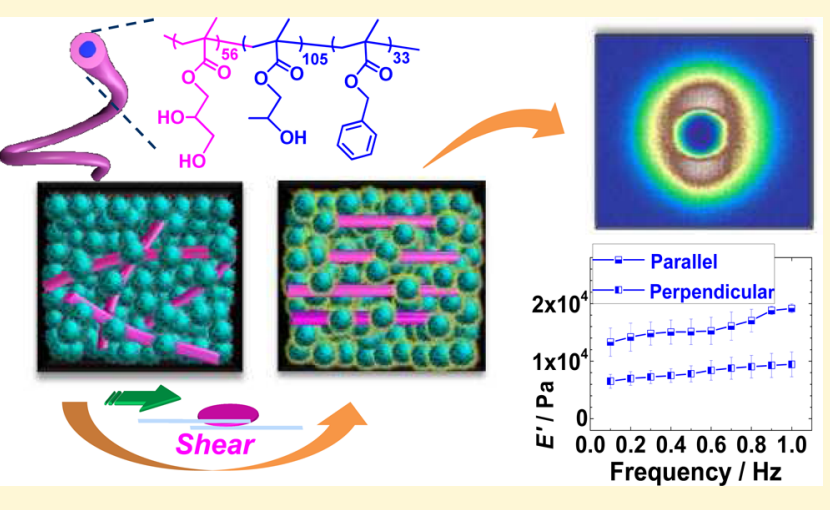
small-angle X-ray scattering as well as modulus and anisotropic swelling experiments. Unexpectedly, the composite gel containing the soft copolymer worms showed the most pronounced anisotropy swelling. The copolymer worms enabled higher RLP loadings than was possible for the stiffer RLPs. For fixed RLP loading, the extent of anisotropic swelling increased with intra-RLP bonding strength. The facile and versatile approach to anisotropic gel construction demonstrated herein is expected to enable new applications for strain sensing or biomaterials for soft tissue repair.

\section{INTRODUCTION}

A major challenge for hydrogel research is the design of hydrogels with controlled morphology over a range of length scales. While many new hydrogels have been reported with excellent mechanical properties, ${ }^{1-10}$ there have been relatively few reports involving anisotropic composite gels. ${ }^{11,12}$ Such gels are gaining increasing attention in view of their potential applications in photonics, ${ }^{13}$ as well as biomaterials that mimic the extra-cellular matrix and direct tissue growth. ${ }^{14}$ Cells can sense and respond to nanoscale textures ${ }^{15}$ which provides the opportunity to guide repair of tissues such as artificial muscles, ${ }^{16}$ tendons, ${ }^{17,18}$ heart tissues, ${ }^{19}$ and neural path- ways $^{14,15,18,20-22}$ and within the spinal cord. ${ }^{23}$ Hydrogels containing aligned rod-like particles (RLPs) are a potential route to anisotropic gels for such applications. Unfortunately, a facile and versatile method for permanently aligning RLPs within hydrogels is currently lacking. Usually, RLP alignment is achieved using high shear, ${ }^{24,25}$ cold drawing, ${ }^{26}$ or solvent evaporation, ${ }^{27}$ which constrain experimental versatility and the potential to include cells. High shear rates are known to

Received: January 11, 2017

Revised: February 14, 2017

Published: February 18, 2017 
Scheme 1. Preparation of Aligned Doubly-Crosslinked NG/RLP Gel Composites (DX NG NLP $\left._{1-x}\right)^{a}$

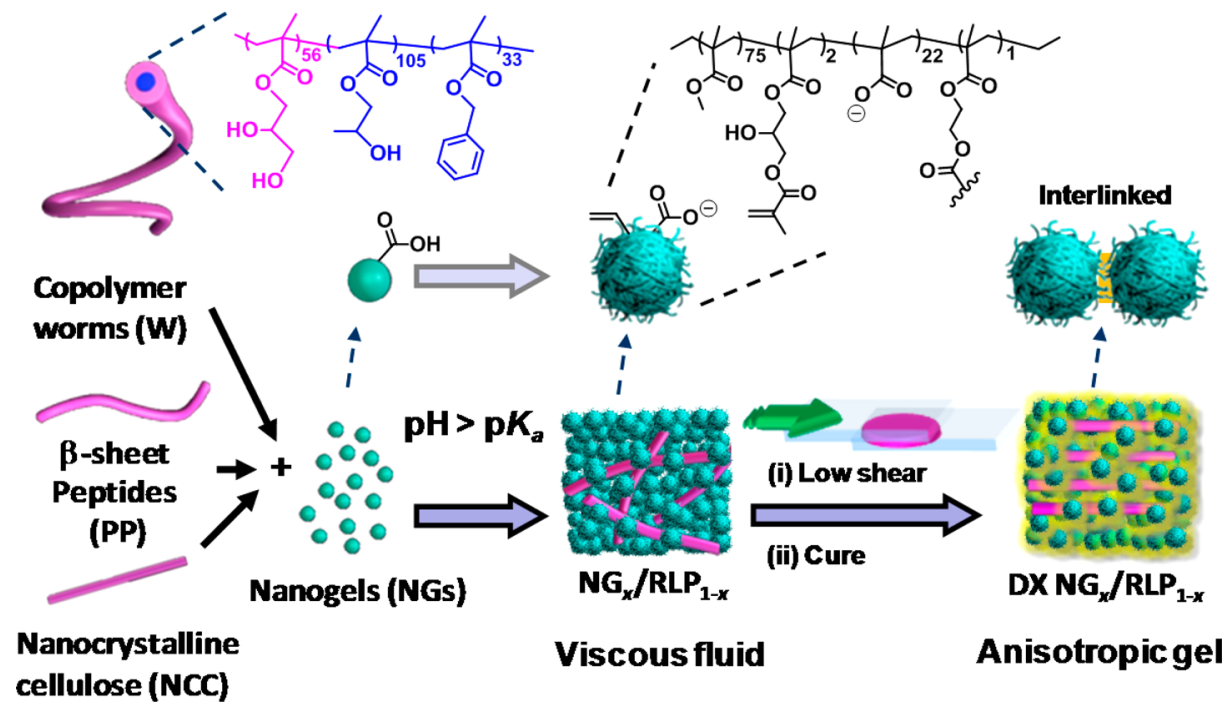

${ }^{a}$ The RLPs were copolymer worms comprised of poly(glycerol monomethacrylate)- $b$-poly(2-hydroxypropyl methacrylate)- $b$-poly(benzyl methacrylate), $\beta$-sheet peptide fibers, or nanocrystalline cellulose fibers. The NGs comprised poly(methyl methacrylate-co-methacrylic acid-coethyleneglycol dimethacrylate) and were vinyl functionalized. A viscous fluid formed when the $\mathrm{pH}$ of mixed $\mathrm{NG}_{x} / \mathrm{RLP}_{1-x}(x=\mathrm{dry} \mathrm{NG}$ weight fraction) dispersions was increased. The latter was sheared, and the NGs were covalently interlinked to form an anisotropic composite gel.

decrease cell viability. ${ }^{28}$ Other methods for obtaining anisotropic gels include freeze-casting, ${ }^{29}$ imprinting, ${ }^{17}$ concentration via dialysis, ${ }^{18}$ magnetic fields, ${ }^{30}$ or self-assembly. ${ }^{14,21,31,32}$ These methods either require conditions that are not cyto-compatible or involve specialized materials and equipment. Alignment methods involving low shear rates provide enhanced application potential with cells. Here, we investigate a new approach for anisotropic composite gel formation that is versatile and scalable and uses inexpensive laboratory equipment. We hypothesized that low shear could be used to align RLPs if they were dispersed in a mixture of soft (swollen) copolymer nanoparticles and that this alignment could be "locked in" if the nanoparticles could be covalently interlinked to form a network. Herein, we investigate anisotropic gels containing RLPs assembled using nanogels (NGs). NGs are cross-linked pH-responsive copolymer nanoparticles with a size less than $100 \mathrm{~nm}$ that swell when the $\mathrm{pH}$ approaches the $\mathrm{p} K_{\mathrm{a}}$ of the constituent polymer. ${ }^{33}$ These nanosized particles are smaller than their larger microgel cousins. ${ }^{34-37}$ NGs have a range of delivery and diagnostics applications. $^{38}$

Three RLP systems were used in this study: soft triblock copolymer worms (W), stiff $\beta$-sheet peptide fibers (PP), and ultrahigh modulus nanocrystalline cellulose (NCC). The copolymer worms were the most flexible, whereas NCC fibers were highly rigid and the PP fibers possessed intermediate stiffness. The shear-induced alignment of small molecule surfactant worms in the presence and absence of spheres has been studied. ${ }^{39-41}$ Those worms were "living" systems because the characteristic time for worm breaking $\left(\tau_{\text {break }}\right)$ was comparable to the experimental shear time $\left(\tau_{\text {shear }}\right)$. Mixtures of such surfactant worms with monomers commonly used to prepare hydrogels are unstable. ${ }^{41}$ Furthermore, orienting such worms within hydrogels is not currently possible to our knowledge. We overcame this obstacle by mixing non-living RLPs with NG macro-cross-linkers to construct anisotropic composite gels. The RLPs used here were less susceptible to breaking and the NGs acted as nanometer-scale gel building blocks. Triblock copolymer worms were the primary RLP system studied (Scheme 1). PP fibers and NCC were used to study the effects of intra-RLP bonding on anisotropic gel swelling and demonstrate versatility of our new, low-shear approach for preparing anisotropic gels.

To align RLPs under shear, the local shear rate must be high compared to the RLPs rotational relaxation time. ${ }^{42}$ The latter is determined by the RLP dimensions as well as colloidal and hydrodynamic interactions and the viscosity of the sheared fluid. Key parameters controlling the hydrodynamic interactions of mixed dispersions of rods and spheres are the ratio of the rod length $(L)$ to the sphere diameter $\left(D_{\mathrm{NG}}\right)$, i.e., $\xi=(L /$ $\left.D_{\mathrm{NG}}\right)$. The ratio of the RLP diameter $\left(D_{\mathrm{RLP}}\right)$ to the sphere diameter $\left(q=\left(D_{\mathrm{RLP}} / D_{\mathrm{NG}}\right)\right)$ is also important. If $\xi \gg 1$, then $q$ determines the nature of the hydrodynamic interactions. ${ }^{39}$ We selected RLPs with large $\xi$ values and used NGs to achieve $q$ values close to unity. This combination provided efficient stress transfer from the NGs to the RLPs and enabled alignment at low shear. Furthermore, because the NGs scattered light weakly, they provided a transparent matrix which enabled RLP alignment to be probed using polarized light optical microscopy.

The three RLP systems examined (Scheme 1) have different types of intra-RLP bonding and hence $\tau_{\text {break }}$ values. Block copolymer worms are soft, flexible RLPs that have attracted considerable interest. ${ }^{43-48}$ They can be conveniently synthesized by polymerization-induced self-assembly (PISA) ${ }^{45,49}$ and are biocompatible. ${ }^{46}$ The worms used for this study comprised a poly(glycerol monomethacrylate)- $b$-poly(2-hydroxypropyl methacrylate)- $b$-poly(benzyl methacrylate) (PGMA $6^{-}$ $\mathrm{PHPMA}_{105}-\mathrm{PBzMA}_{33}$ ) triblock copolymer synthesized directly in water using PISA. ${ }^{50}$ Concentrated worm gels have relatively low moduli ${ }^{46}$ as a result of their relatively small $\tau_{\text {break }}$ values. Herein, we report for the first time their incorporation into composite gels as well as their alignment. In contrast to worms, $\beta$-sheet peptide fibers self-assemble owing to the attractive intermolecular H-bonding between the backbone amides of PP chains and $\pi-\pi$ interactions of aromatic residues. ${ }^{51}$ PP gels 
have a range of applications which include substrates for cell culture. $^{51-53}$ They have also been used to form composites with temperature-responsive microgels. ${ }^{54} \mathrm{PP}$ fiber dispersions form relatively brittle gels at low concentration. ${ }^{55,56}$ Accordingly, PP physical gels have higher $\tau_{\text {break }}$ values than worms. NCC fibers, which are obtained from a renewable, biocompatible, ${ }^{57}$ and abundant material, exhibit ultrahigh moduli ${ }^{58,59}$ (i.e., $\left.150 \mathrm{GPa}^{60}\right)$, have been used to reinforce conventional gels, ${ }^{61}$ and can form hierarchical structures. ${ }^{62}$ NCC fibers do not break down under normal rheological experimental time frames and so were selected in this study as an RLP system with an effectively infinite $\tau_{\text {break }}$ value. These RLPs enabled us to study the effect of $\tau_{\text {break }}$ value on gel properties.

We recently established a method for preparing isotropic hydrogels using $\mathrm{pH}$-responsive vinyl-functionalized $\mathrm{NGs}$ as macro-cross-linkers. ${ }^{33}$ Concentrated NG dispersions were prepared using a convenient scalable synthesis and formed space-filling physical gels upon raising the solution $\mathrm{pH}$. These gels formed due to NG particle swelling and were covalently interlinked via peripheral vinyl groups to form $\mathrm{pH}$-responsive hydrogels, which are termed doubly cross-linked NGs (DX NGs). ${ }^{33}$ In the present study, the NGs played two crucial roles in the formation of anisotropic DX NG/RLP gels (Scheme 1): they transferred shear stress to the RLPs and also enabled the otherwise transient anisotropy generated under shear to be permanently locked in place.

In this paper, we first characterize the NG and copolymer worm binary mixtures. Shear-induced alignment of the worms and the formation of anisotropic DX NG/worm gels is then demonstrated using a new rheo-optical technique, small-angle $\mathrm{X}$-ray scattering (SAXS), and anisotropic swelling studies. The DX NG/worm gels are shown to exhibit low cytotoxicity which is encouraging for future soft tissue repair applications. The versatility of our approach is then demonstrated using PPs and NCCs. Dynamic mechanical analysis data for the DX NG/ NCC system demonstrate the presence of anisotropic modulus values. The aligned composite gels are studied using polarized light microscopy as well as $\mathrm{pH}$-triggered swelling, and the relationship between intrarod bonding and anisotropic swelling is discussed. The new and versatile anisotropic gel assembly established here may lead to optical sensors using polarized light due to the strong correlation between birefringence and RLP orientation ${ }^{63,64}$ or next-generation gels for directing tissue growth in vivo.

\section{EXPERIMENTAL SECTION}

Materials. Methyl methacrylate (MMA, 98.5\%), methacrylic acid (MAA, 99\%), ethylene glycol dimethacrylate (EGDMA, 98\%), benzyl methacrylate (BzMA, 96\%), glycidyl methacrylate (GM, 97\%), ammonium persulfate (APS, 98\%), sodium dodecyl sulfate (SDS, 98.5\%), $N, N, N^{\prime}, N^{\prime}$-tetramethylethylenediamine (TEMED, 99\%), and microcrystalline cellulose $(20 \mu \mathrm{m})$ were purchased from Aldrich and used as received. Doubly distilled deionized water was used for all experiments. Glycerol monomethacrylate (GMA; 99.8\%) was donated by GEO Specialty Chemicals (Hythe, UK) and used without further purification. 2-Hydroxypropyl methacrylate (HPMA, 97\%) and 4,4'azobis(4-cyanopentanoic acid) (ACVA; V-501; 99\%) were purchased from Alfa Aesar (Heysham, UK) and used as received. 2-Cyano-2propyl dithiobenzoate (CPDB, $80 \%$ from ${ }^{1} \mathrm{H}$ NMR spectroscopy) was purchased from Strem Chemicals (Cambridge, UK). Tris(2carboxyethyl)phosphine (TCEP hydrochloride, 99\%) was purchased from Amresco (Solon, Ohio, USA). $\mathrm{CD}_{3} \mathrm{OD}$ and dimethyl sulfoxide- $d_{6}$ (DMSO) were purchased from Goss Scientific (Nantwich, UK). All other solvents were purchased from Fisher Scientific (Loughborough, $\mathrm{UK})$ and used as received.
Synthesis of Vinyl-Functionalized Nanogels (NGs). The NG particles were prepared via monomer-starved emulsion polymerization using a method described elsewhere ${ }^{33}$ (see Scheme S1a). Briefly, SDS $(1.2 \mathrm{~g}, 4.0 \mathrm{mmol})$ was dissolved in water $(240 \mathrm{~g})$, and the solution was purged with nitrogen before addition of APS $(0.20 \mathrm{~g}, 0.90 \mathrm{mmol})$ in water $(2.0 \mathrm{~mL})$. A monomer feed comprising MMA (42.0 g, 0.42 $\mathrm{mol})$, MAA (10.0 g, $0.12 \mathrm{~mol})$, and EGDMA (1.1 g, $5.0 \mathrm{mmol})$ was added at a rate of $0.30 \mathrm{~mL} \mathrm{~min}^{-1}$. The product was extensively dialyzed against water. For vinyl functionalization, GM (4.50 g, 0.39 $\mathrm{mol}$ ) was added to the NG dispersion ( $120 \mathrm{~g}, 5.0 \mathrm{wt} \%)$ and the $\mathrm{pH}$ was adjusted to 5.1 . The dispersion was stirred for $8 \mathrm{~h}$ at $40{ }^{\circ} \mathrm{C}$. The dispersion was repeatedly washed with $n$-hexane, and residual solvent was removed by rotary evaporation.

Synthesis of PGMA ${ }_{56}$. A protocol for the synthesis of PGMA macro-CTA (chain transfer agent) is given in Scheme S1b. To a round-bottomed flask containing CPDB RAFT agent $(6.03 \mathrm{~g}, 0.020$ $\mathrm{mol})$, GMA monomer $(203 \mathrm{~g}, 1.27 \mathrm{~mol})$ and anhydrous ethanol (156 $\mathrm{g}, 3.38 \mathrm{~mol}$ ) were added to afford a target degree of polymerization (DP) of 63. ACVA initiator (1.14 g, $4.07 \mathrm{mmol}$, CTA/ACVA molar ratio $=5.0$ ) was added, and the resulting pink solution was purged with $\mathrm{N}_{2}$ for $20 \mathrm{~min}$, before the sealed flask was immersed into an oil bath set at $70{ }^{\circ} \mathrm{C}$. After $140 \mathrm{~min}$ (69\% conversion as judged by ${ }^{1} \mathrm{H}$ NMR), the polymerization was quenched by immersion of the flask in an ice bath followed by exposure to air. The crude polymer was purified by precipitation into excess $\mathrm{CH}_{2} \mathrm{Cl}_{2}$ and washed three times in this solvent before being dried under high vacuum at $40{ }^{\circ} \mathrm{C} .{ }^{1} \mathrm{H}$ NMR analysis indicated a DP of 56 (see Figure S1). DMF GPC analysis (Figure S2) indicated $M_{\mathrm{n}}$ and $M_{\mathrm{w}} / M_{\mathrm{n}}$ values of $1.43 \times 10^{4} \mathrm{~g} \mathrm{~mol}^{-1}$ and 1.14 , respectively.

Synthesis of PGMA 56 -PHPMA ${ }_{105}-$ PBzMA $_{33}$ Triblock Copolymer. The method used is depicted in Scheme S1b. PGMA 56 macroCTA $(4.28 \mathrm{~g}, 0.28 \mathrm{mmol})$ was added to a $100 \mathrm{~mL}$ round-bottomed flask, followed by HPMA monomer $(0.25 \mathrm{~g}, 0.03 \mathrm{~mol})$ and water $(39.0$ $\mathrm{g}$, to make a $15 \% \mathrm{w} / \mathrm{w}$ solution). ACVA was then added ( $0.02 \mathrm{~g}, 0.07$ $\mathrm{mmol}$, CTA/ACVA molar ratio $=4.0$ ), and the solution was purged with $\mathrm{N}_{2}$ for $30 \mathrm{~min}$. The sealed flask was immersed in an oil bath set at $70{ }^{\circ} \mathrm{C}$. The solution was magnetically stirred for $2.5 \mathrm{~h}$ to ensure complete monomer conversion ( $>99 \%$ as judged by ${ }^{1} \mathrm{H}$ NMR in $\mathrm{CD}_{3} \mathrm{OD}$ ), and the polymerization was quenched by cooling the flask to $20{ }^{\circ} \mathrm{C}$ and exposure to air. BzMA monomer (1.64 g, $\left.9.33 \mathrm{mmol}\right)$ was then immediately added along with additional ACVA $(20.0 \mathrm{mg}$, $0.07 \mathrm{mmol}$ ) before the reaction mixture was degassed with $\mathrm{N}_{2}$ for a further $30 \mathrm{~min}$. The flask was then replaced in the oil bath at $70{ }^{\circ} \mathrm{C}$ for a further $5 \mathrm{~h}$, and the second-stage BzMA polymerization was quenched by exposure to air while at $20{ }^{\circ} \mathrm{C}$. A sample of the copolymer dispersion was freeze-dried and dissolved in DMSO for ${ }^{1} \mathrm{H}$ NMR analysis (see Figure S1), which indicated that essentially all of the BzMA monomer had been incorporated into the copolymer. DMF GPC analysis indicated $M_{\mathrm{n}}=30600 \mathrm{~g} \mathrm{~mol}^{-1}$ and $M_{\mathrm{w}} / M_{\mathrm{n}}=1.13$ for the PGMA $_{56}-$ PHPMA $_{105}$ diblock copolymer and $M_{\mathrm{n}}=33200 \mathrm{~g} \mathrm{~mol}^{-1}$ and $M_{\mathrm{w}} / M_{\mathrm{n}}=1.13$ for $\mathrm{PGMA}_{56}-\mathrm{PHPMA}_{105}-\mathrm{PBzMA}_{33}$ triblock copolymer (see Figure S2).

Preparation of Self-Assembled Peptide Fibers (PP). The peptide studied was FEFEFKFE (F for phenylalanine, E for glutamate, and $\mathrm{K}$ for lysine residues), which was purchased from BIOMATIK Corporation (Delaware, USA) with more than $95 \%$ purity. Its identity and purity were confirmed in-house by ESI-MS and reverse phase HPLC, respectively. To prepare fibers, the peptide (19.4 mg, 7.0 $\mu \mathrm{mol})$ was dissolved in HPLC-grade water $(700 \mu \mathrm{L})$ by sonication for $30 \mathrm{~min}$ at $80 \mathrm{kHz}$. The peptide solution $(\mathrm{pH} 1.8)$ was titrated using 0.5 $\mathrm{M} \mathrm{NaOH}$ to trigger gelation. A hydrogel was obtained at $\mathrm{pH}$ 5.3. The gel volume was adjusted to $1.0 \mathrm{~mL}$ using water to obtain a $14 \mathrm{mM}$ $(\sim 1.9 \% \mathrm{w} / \mathrm{v})$ hydrogel concentration. The gel was allowed to equilibrate overnight at $4{ }^{\circ} \mathrm{C}$ and used within $24 \mathrm{~h}$.

Preparation of Nanocrystalline Cellulose. NCC was prepared by acid treatment of microcrystalline cellulose using a literature protocol. ${ }^{65}$ Briefly, microcrystalline cellulose $(5.0 \mathrm{~g})$ was mixed with water $(18 \mathrm{~g})$, and the resulting suspension was placed in an ice bath. Sulfuric acid ( $32 \mathrm{~g}$ of $98 \%$ solution) was added slowly, and the mixture stirred for $150 \mathrm{~min}$ at $45^{\circ} \mathrm{C}$. [Caution: Concentrated acids are corrosive.] 
The product was purified by repeated centrifugation and redispersion in water.

Preparation of Anisotropic Doubly Cross-Linked Gel Composites. The NG and worm (W) proportions in the gel composites are reported as $\mathrm{DX} \mathrm{NG} / \mathrm{W}_{1-x}$, where $x$ is the dry weight fraction of NG used to prepare each sample. The following gives the preparation protocol for $\mathrm{DX} \mathrm{NG}_{0.5} / \mathrm{W}_{0.5}$, which was prepared at a particle concentration of $12 \mathrm{wt} \%$ at $\mathrm{pH} 7.5$ by vortex-mixing a NG dispersion $(0.70 \mathrm{~g}, 14.7 \mathrm{wt} \%)$ with an aqueous worm dispersion $(0.60$ $\mathrm{g}, 17.1 \mathrm{wt} \%)$ and water $(0.285 \mathrm{~g})$. An APS solution $(60 \mu \mathrm{L}, 78 \mathrm{mM})$ was added followed by addition of an alkaline TEMED solution (70 $\mu \mathrm{L}$ ). The latter was prepared by mixing $4.0 \mathrm{M} \mathrm{NaOH}$ with TEMED at a volume ratio of $24: 1$. The $\mathrm{pH}$ increase produced a physical gel containing singly cross-linked NGs, denoted $\mathrm{NG}_{0.5} / \mathrm{W}_{0.5}$. For DX NG/ worm composites with different $x$ values, the masses of NG and worms were adjusted and the amounts of APS and $\mathrm{NaOH} / \mathrm{TEMED}$ changed in proportion to the NG content. Unless otherwise stated, the total copolymer content (worms and/or NG) was $12.0 \mathrm{wt} \%$. Anisotropic DX NG/worm composites were prepared by aligning worms during in situ cross-linking of the physical gels. Rotational shear or linear shear was used with either planar or cylindrical geometries. For the former, an Anton Paar Physica MCR-301 rheometer equipped with a SIPLI device was employed (see below). A shear rate of $0.1 \mathrm{~s}^{-1}$ and a gap of $200 \mu \mathrm{m}$ were used. The temperature employed was $45^{\circ} \mathrm{C}$.

In the case of linear shear, the $\mathrm{NG}_{x} / \mathrm{W}_{1-x}$ precursor physical gel was placed between two microscope slides fixed to the clamps of a World Precision Instruments (AL-1000) syringe pump (see Figure S3). The gap between the two microscope slides was $500 \mu \mathrm{m}$, and the shear rate was $0.1 \mathrm{~s}^{-1}$. A heated air flow was carefully directed over the top of the uppermost microscope slide to give a surface temperature of $\sim 45^{\circ} \mathrm{C}$, and care was taken to minimize gel dehydration. Using the same protocol, anisotropic $\mathrm{DX} \mathrm{NG} / \mathrm{PP}_{1-x}$ gel composites were prepared by mixing $0.15 \mathrm{~g}$ of PP fibers ( $2 \mathrm{wt} \%)$ with NG ( $440 \mu \mathrm{L}, 22 \mathrm{wt} \%)$. Then, APS $(35 \mu \mathrm{L}, 78 \mathrm{mM})$ was added, followed by $\mathrm{NaOH} / \mathrm{TEMED}$ solution $(30 \mu \mathrm{L})$. DX NG $\mathrm{N}_{0.92} / \mathrm{NCC}_{0.08}$ was prepared by mixing NCC $(0.50 \mathrm{~g}$ of $2 \mathrm{wt} \%)$ with NG ( $440 \mu \mathrm{L}, 22 \mathrm{wt} \%)$, followed by addition of APS $(35 \mu \mathrm{L}, 78 \mathrm{mM})$ and NaOH/TEMED $(30 \mu \mathrm{L})$ solution. The mixtures were vortexed for $2 \mathrm{~min}$ prior to application of shear.

For shear alignment using a cylindrical geometry, strings of DX $\mathrm{NG}_{0.5} / \mathrm{W}_{0.5}$ were prepared by injecting the $\mathrm{NG}_{0.5} / \mathrm{W}_{0.5}$ precursor through a syringe needle (Gauge 18) onto a glass slide using a shear rate of $18 \mathrm{~s}^{-1}$. Such strings were cured in a sealed Petri dish at $37^{\circ} \mathrm{C}$ for $1 \mathrm{~h}$.

Isotropic gel composites were prepared by placing the physical gel in an O-ring sealed between two microscope glass slides. The gels were cured at $37{ }^{\circ} \mathrm{C}$ overnight. Several control DX NG gels were also prepared using the same approach without the addition of copolymer worms (see Figure S5e,f).

Physical Measurements. Molecular weight data were obtained using a DMF GPC setup using poly(methyl methacrylate) calibration standards, as described earlier. ${ }^{66}{ }^{1} \mathrm{H}$ NMR spectroscopy was performed using a $400 \mathrm{MHz}$ Bruker Avance-500 spectrometer. Potentiometric titration data were obtained in the presence of $0.05 \mathrm{M} \mathrm{NaCl}$ using a Mettler Toledo titrator. Dynamic light scattering (DLS) and electrophoretic mobility data were obtained using a Malvern Zetasizer NanoZS instrument and buffer solutions. A Hitachi U-1800 spectrophotometer was used for UV-visible spectroscopy measurements. An Olympus BX41 microscope was used to obtain optical microscopy images using transmitted light. For polarized light optical microscopy (POM), the light was passed through a polarizer and an analyzer, with the latter fixed at $90^{\circ}$ with respect to the former. SEM and TEM images were obtained using Philips FEGSEM at $6 \mathrm{kV}$ and Phillips CM100 at $100 \mathrm{kV}$ instruments, respectively. AFM studies of the DX NG/worm composites were performed using a Bruker Catalyst (mounted on a Nikon Eclipse Ti inverted light microscope) with a Nanoscope V controller. Imaging was performed in ScanAsyst mode in liquid using ScanAsyst Fluid cantilevers (nominal spring constant, $0.7 \mathrm{~nm} / \mathrm{V}$; nominal probe radius, $20 \mathrm{~nm}$ (Bruker AXS, SAS)). For pure PP and NCC fibers, ScanAsyst mode in air was used.
Images were second-order flattened using the Bruker Nanoscope Analysis software.

SAXS data were obtained using a modified Bruker AXS Nanostar instrument equipped with a microfocus Xenocs Genix 3D X-ray source ( $\mathrm{Cu} \mathrm{K} \alpha$ radiation), a collimator comprised of two sets of motorized scatterless slits, a camera length of $1.46 \mathrm{~m}$, and a 2D HiSTAR multiwire gas detector (pixel size, $0.105 \mathrm{~mm}^{2}$ ). 2D SAXS patterns were collected from sample films ( $500 \mu \mathrm{m}$ thickness) sealed between two mica disks $(25 \mu \mathrm{m}$ thickness each). The scattering data were reduced to $1 \mathrm{D}$ plots by Nika SAS macros for Igor Pro. ${ }^{67}$

Shear-induced polarized light imaging (SIPLI) measurements were conducted using a prototype rheometer for mechano-optical rheology. A mechanical Anton Paar Physica MCR 301 rheometer was coupled with an optical SIPLI device based on principles of reflection polariscopy. ${ }^{68}$ The optical part of the instrument enabled a polarized light image of the sample loaded in the rheometer to be obtained during shear flow. Samples were placed between two parallel horizontal plates of the rheometer (one of the plates was transparent for light), and a shear pulse was applied by rotating the top plate.

Conventional dynamic rheology measurements were performed using a TA Instruments AR G2 rheometer. A $20 \mathrm{~mm}$ diameter plate geometry was used, and the gap was $2500 \mu \mathrm{m}$. DMA measurements were performed using a TA-Q800 instrument equipped with the shear sandwich (shear) geometry at $25{ }^{\circ} \mathrm{C}$. For these measurements, DX $\mathrm{NG}_{0.92} / \mathrm{NCC}_{0.08}$ samples were prepared by vortexing $\mathrm{NG}(700 \mu \mathrm{L}, 22$ wt \%) and NCC (400 $\mu \mathrm{L}, 3.6 \mathrm{wt} \%)$ followed by addition of APS (55 $\mu \mathrm{L}, 78 \mathrm{mM})$ and $\mathrm{NaOH} / \mathrm{TEMED}(47 \mu \mathrm{L})$, mixing, and curing as described above.

Anisotropic Swelling Experiments. Anisotropic gel swelling was studied by allowing samples to reach swelling equilibrium in buffer solutions over a period of $\sim 18 \mathrm{~h}$. The dimensions for each swollen sample $(W$ and $L)$ were measured at $\mathrm{pH} 8.0$, while dimensions corresponding to the collapsed state $\left(W_{\mathrm{o}}\right.$ and $\left.L_{\mathrm{o}}\right)$ were measured at $\mathrm{pH}$ 5.0. A sketch of the geometries used is shown in Figure 3e. The anisotropic swelling parameter $(A)$ was calculated ${ }^{69}$ using

$$
A=\frac{\alpha_{W}}{\alpha_{L}}-1
$$

where $\alpha_{W}\left(=W / W_{\mathrm{o}}\right)$ and $\alpha_{L}\left(=L / L_{\mathrm{o}}\right)$ are the linear swelling ratios measured perpendicular and parallel to the shear direction, respectively.

Cytotoxicity Studies. The cytotoxicity of $\mathrm{DX} \mathrm{NG}_{0.5} / \mathrm{W}_{0.5}$ was examined in the presence of human nucleus pulposus (NP) cells. The latter were seeded onto 24-well plates at a density of $5 \times 10^{4}$ per well and cultured in Dulbecco's modified Eagle's medium supplemented with $10 \%$ fetal bovine serum (FBS, Gibco), antibiotic/antimycotic, and L-ascorbic acid 2-phosphate $(10 \mu \mathrm{M})$ (Sigma-Aldrich, UK) in a humidified atmosphere containing $5 \% \mathrm{CO}_{2}$ at $37^{\circ} \mathrm{C}$ for $24 \mathrm{~h}$. The DX $\mathrm{NG}_{0.5} / \mathrm{W}_{0.5}$ samples $(20 \mathrm{mg})$ were washed in phosphate buffered saline (PBS) for 2 days prior the biocompatibility test, sterilized with $70 \%$ ethanol, and rinsed with sterile PBS. The gel samples were put into 0.4 $\mu \mathrm{m}$ cell-culture inserts (BD Biosciences, UK) that were placed in the well plate. Cell viability was tested by the MTT assay (Aldrich, UK) after 1, 4, and 7 days. An empty insert was used as the control. For each time point, three data points were collected using a BMG Labtech FLUOstar plate reader.

\section{RESULTS AND DISCUSSION}

Properties of Nanogels, Worms, and Mixed Dispersions. All of the materials used to construct the anisotropic composite gels in this study were prepared using scalable methods. The NGs and copolymer worms were synthesized by emulsion polymerization and RAFT solution polymerization, respectively (Scheme S1). The worms were characterized using ${ }^{1} \mathrm{H}$ NMR spectroscopy and GPC (Figures S1 and S2), and their composition is shown in Scheme 1. The NGs contained $22 \mathrm{~mol}$ $\%$ MAA as well as vinyl groups from GM ( $2 \mathrm{~mol} \%)$ and had an 
apparent $\mathrm{p} K_{\mathrm{a}}$ of $7.1 .^{33}$ Their composition is also shown in Scheme 1.

The NG and worm morphologies were assessed using TEM (Figure 1a,b). The NGs were spheres with a number-average

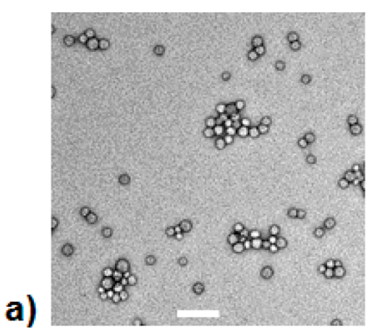

b)
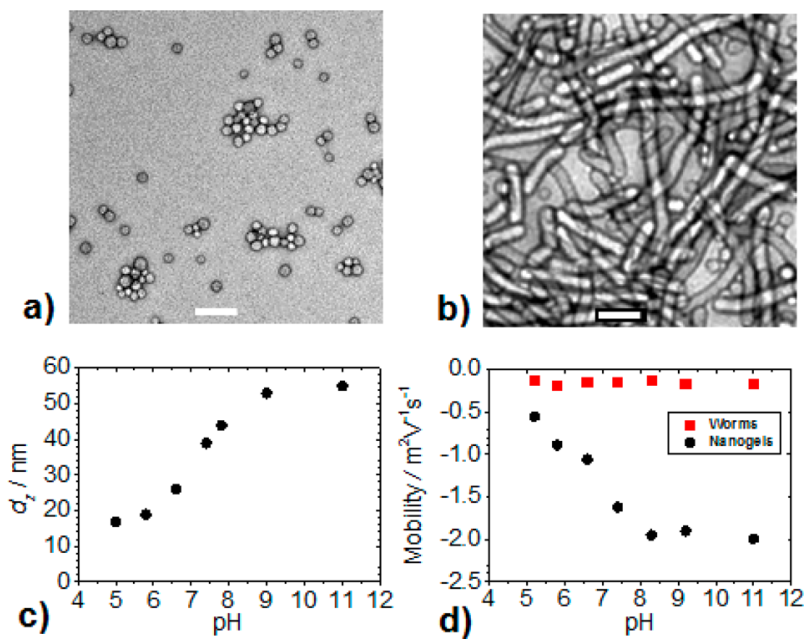

Figure 1. TEM images obtained for (a) NGs and (b) copolymer worms, respectively. The scale bars are $100 \mathrm{~nm}$. (c) Variation of $z$ average diameter with $\mathrm{pH}$ for the NG particles. (d) Electrophoretic mobility vs $\mathrm{pH}$ for the NGs and copolymer worms.

diameter of $17 \pm 3 \mathrm{~nm}$. The anisotropic worms (Figure $1 \mathrm{~b}$ ) had a mean diameter of $35 \pm 5 \mathrm{~nm}$ and a polydisperse length distribution with a number-average worm length of $310 \pm 280$ $\mathrm{nm}$. High worm length polydispersities are typical for such syntheses and arise from the stochastic nature of worm formation via multiple $1 \mathrm{D}$ fusion of precursor spheres. ${ }^{48}$ The NGs were $\mathrm{pH}$-responsive, and the $z$-average DLS diameter $\left(d_{\mathrm{z}}\right)$ strongly increased from $\mathrm{pH} 5$ to $\mathrm{pH} 11$ (Figure 1c). The NGs had a $d_{\mathrm{z}}$ of $17 \mathrm{~nm}$ in the collapsed state $(\mathrm{pH} 5)$ but swelled up to $52 \mathrm{~nm}$ at $\mathrm{pH} 9$. The $d_{\mathrm{z}}$ value was $\sim 40 \mathrm{~nm}$ at $\mathrm{pH} \sim 7.5$ and hence $q \sim 1$, as intended. The latter $\mathrm{pH}$ was used to prepare the composite gels.

One prerequisite for achieving shear-induced worm alignment in NG/RLP binary mixtures is that these two species do not attract each other because NG/RLP aggregate formation would otherwise oppose ordering. Electrophoretic mobility vs $\mathrm{pH}$ studies (Figure 1d) confirmed that the NGs were negatively charged and became more strongly anionic as the $\mathrm{pH}$ approached 7.5. The copolymer worms remained weakly anionic at all $\mathrm{pH}$ values studied. The anionic character observed for the NGs and worms was due to neutralization of MAA repeat units and also residual anionic carboxylate groups from the ACVA initiator, ${ }^{66}$ respectively. Thus, there were no attractive electrostatic interactions between the NGs and worms.

Dilute binary $\mathrm{NG} /$ worm dispersions $\left(\mathrm{NG}_{x} / \mathrm{W}_{1-x}\right.$, where $x$ is the dry weight fraction of $N G$ ) were investigated using turbidity and DLS measurements to confirm that both components remained colloidally stable when mixed. Digital photographs of the sample vials (Figure S4a) did not show any evidence of visible aggregates. The optical density measured at $400 \mathrm{~nm}$ of the binary mixtures (Figure S4b) followed a linear relationship with $x$, indicating attractive interactions were not present between the NGs and worms. The absence of attractive interactions was also shown by DLS data (Figure S4c), which reported bimodal size distributions due to each (isolated) component. In summary, the NGs and worms were both well dispersed in the mixed dispersions.

Low Shear Alignment and Assembly of Anisotropic DX NG/Worm Gels. A preliminary investigation was conducted of the ability of binary NG/worm dispersions to form isotropic covalent gels and to determine the compositional limits of $\mathrm{DX} \quad \mathrm{NG}_{x} / \mathrm{W}_{1-x}$ systems (see Supporting Discussion and Figure S5). Briefly, isotropic gels could be formed provided $x$ was greater than or equal to 0.35 , which was identified from rheology data as the gel-to-fluid boundary. Whereas the DX NGs were covalently interlinked, the worms relied upon hydrophobic interactions and contributed less to the composite gel modulus. The $\tau_{\text {break }}$ value for the worms was identified as 1.0 from dynamic rheology data (Figure S6). MTT data obtained for nucleus pulposus cells in the presence of DX $\mathrm{NG}_{0.5} / \mathrm{W}_{0.5}$ composite gel were indistinguishable from a gel-free control (Figure S7) which indicated that significant release of toxic components did not occur over a period of 7 days.

Shear-induced worm alignment was investigated using a modified rheometer incorporating SIPLI, which enables in situ monitoring of the sample under shear (see Experimental Section). ${ }^{78}$ Figure 2 shows polarized light images obtained for

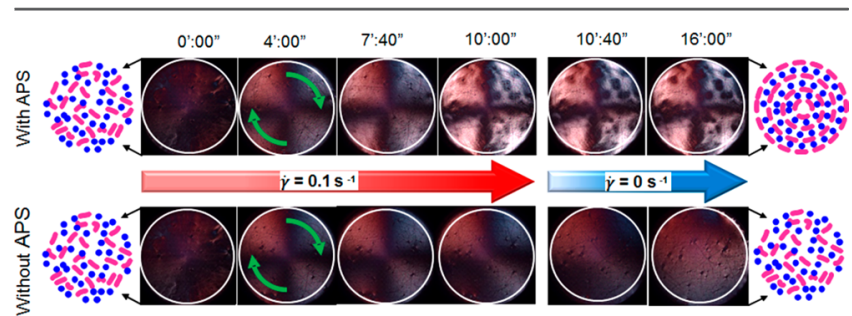

Figure 2. Rotational shear $(\gamma)$ was applied to $\mathrm{NG}_{0.5} / \mathrm{W}_{0.5}$ binary mixtures while being viewed with polarized light using SIPLI. The samples were heated to $45^{\circ} \mathrm{C}$, and the shear was terminated after 10 $\mathrm{min}$. The top row shows the sample that underwent double crosslinking (and formed $\mathrm{DX} \mathrm{NG} \mathrm{NG}_{0.5} / \mathrm{W}_{0.5}$ ) during shear due to APS. The bottom row shows the control sample that did not contain APS. The green arrows indicate the direction of shear, and the cartoons depict the proposed initial and final composite gel morphologies.

two $\mathrm{NG}_{0.5} / \mathrm{W}_{0.5}$ physical gels. A mixed dispersion (top row) contained added APS initiator. The other control dispersion (bottom row) did not contain APS. Both binary gels were heated to $45{ }^{\circ} \mathrm{C}$, and characteristic Maltese cross patterns were observed under shear, indicating uniaxial alignment. ${ }^{70-72}$ After $10 \mathrm{~min}$, the shear was terminated. The now doubly cross-linked APS-loaded gel retained the Maltese cross. In contrast, the Maltese cross rapidly disappeared for the control sample after shear was terminated. The NGs aligned the worms when sheared in both cases, but covalent interlinking of NG particles was required to "lock in" this alignment once shear was terminated. The NGs enabled the worms to be aligned at very low shear rates $\left(\sim 0.1 \mathrm{~s}^{-1}\right.$ at the sample edge $)$. These results contrast to other studies which have required much higher shear rates (i.e., $200 \mathrm{~s}^{-1}$ ) to align anisotropic particles in gels. ${ }^{25,73}$ In the present study, the ability to align the worms at low shear is attributed to the formation of a space-filling physical gel due to $\mathrm{pH}$-triggered NG particle swelling and the mutual repulsion between the two colloidal components with comparable diameters (i.e., $q \sim 1$ ).

Building on the above results, we developed a simple linear shear method for aligning worms within DX NG/worm gels using inexpensive laboratory equipment (see Figure S3). $\mathrm{NG}_{x} /$ 

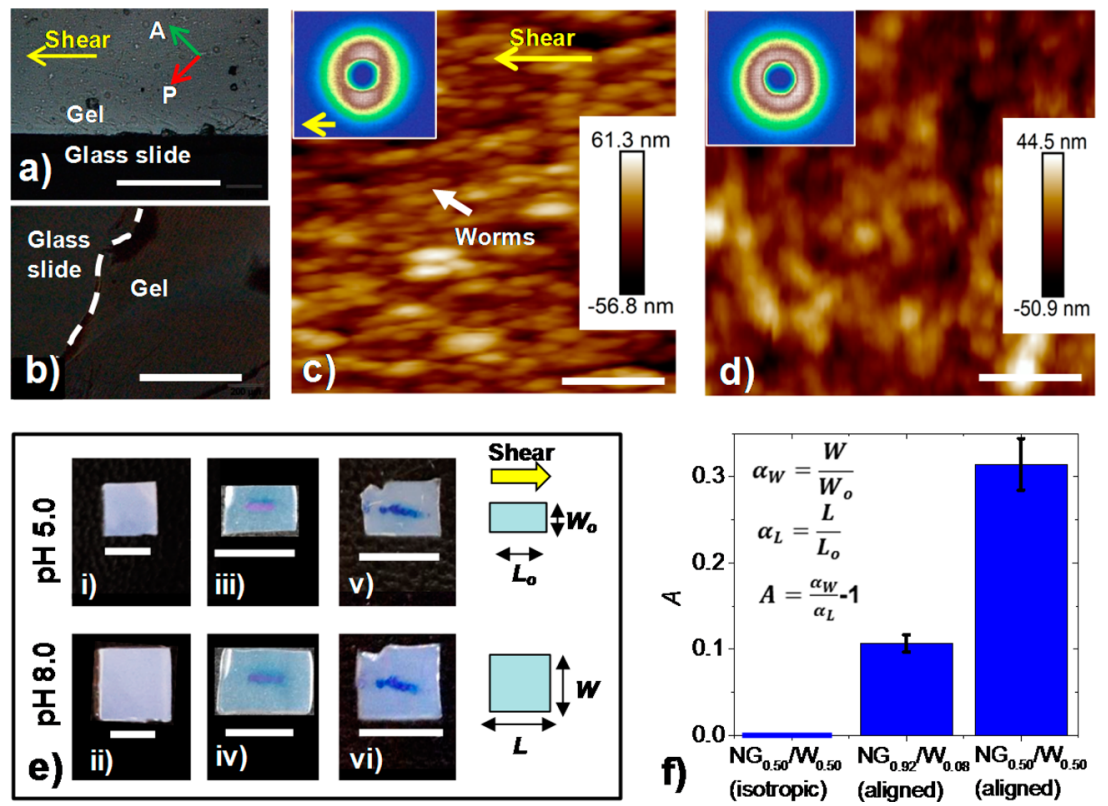

Figure 3. POM images of $\mathrm{DX} \mathrm{NG}_{0.5} / \mathrm{W}_{0.5}$ gels prepared using (a) linear shear and (b) without shear. $\mathrm{A}$ and $\mathrm{P}$ in (a) are the directions of the analyzer and polarizer. (c, d) AFM images obtained for anisotropic and isotropic $\mathrm{DX} \mathrm{NG}_{0.5} / \mathrm{W}_{0.5}$ composites in water, respectively. 2D SAXS patterns for each film are shown as insets. The shear direction for the inset in (c) is indicated. (e) Isotropic $D X \mathrm{NG}_{0.5} / \mathrm{W}_{0.5}\left(\mathrm{i}\right.$, ii), anisotropic $\mathrm{DX} \mathrm{NG}_{0.92} / \mathrm{W}_{0.08}$ films (iii, iv), and anisotropic $\mathrm{DX} \mathrm{NG} \mathrm{N}_{0.5} / \mathrm{W}_{0.5}$ (v, vi) films at pH 5.0 or 8.0. Blue marker pen lines were used to record the shear directions. Scale bars: (a, b) $400 \mu \mathrm{m}$; (c, d) $200 \mathrm{~nm}$; (e) $6.0 \mathrm{~mm}$. The cartoon depicts the anisotropic swelling. (f) Anisotropic swelling parameters $(A)$ from the images shown in $(\mathrm{e})$.

$\mathrm{W}_{1-x}$ physical gels were subjected to planar shear $\left(0.1 \mathrm{~s}^{-1}\right)$ and simultaneously cured at $\sim 45{ }^{\circ} \mathrm{C}$. A polarized light optical microscopy (POM) image (Figure 3a) shows a bright area due to shear-aligned worms within the $\mathrm{DX} \mathrm{NG}_{0.5} / \mathrm{W}_{0.5}$ gel. A control (isotropic) DX $\mathrm{NG}_{0.5} / \mathrm{W}_{0.5}$ gel that had not been subjected to shear is also shown (Figure $3 \mathrm{~b}$ ) for comparison. In that case, bright areas were not observed in any orientation, indicating that no alignment had occurred. The anisotropic DX $\mathrm{NG}_{0.5} / \mathrm{W}_{0.5}$ gels were examined using AFM (Figure $3 \mathrm{c}$ ). Worm alignment in the direction of shear and an anisotropic morphology are evident. As a control, a nonaligned DX $\mathrm{NG}_{0.5} / \mathrm{W}_{0.5}$ control sample was examined using AFM (Figure 3d). The film showed randomly dispersed nano-objects and was isotropic. These data confirm that shear-induced alignment was achieved for planar DX NG/worm films using low shear.

SAXS is an ideal method for probing anisotropy because it is statistically robust and reports averaged structural information via scattering. 2D SAXS patterns were obtained for $\mathrm{DX} \mathrm{NG}_{0.5} /$ $\mathrm{W}_{0.5}$ films where the incident beam was perpendicular to the film plane. An anisotropic film (inset of Figure 3c) and a control isotropic film (inset of Figure 3d) were examined. Only the former gave a $2 \mathrm{D}$ pattern that was clearly elongated (Figure $3 c)$, confirming worm alignment. The 2D SAXS patterns were reduced to $1 \mathrm{D}$ profiles both parallel and perpendicular to the direction of shear (see Figure S8). The SAXS data for both the aligned and isotropic films have intensity minima at $q_{\min } \sim$ $0.028 \AA^{-1}$ associated with the first minimum of the worm form factor and suggest that the radius of the worm core crosssection $R_{\mathrm{w}}$ was about $13.5 \mathrm{~nm}$ (using $q_{\min } R_{\mathrm{w}}=3.83^{74}$ ). A higher scattering intensity was observed for the low $q$ region for the aligned film which indicates the preferred orientation of the worms along the shear direction (Figure S8c). In contrast, there was no difference between the scattering profiles within the film plane for the isotropic, nonaligned film (Figure S8d).
Because our composites consisted of aligned (anisotropic) worms that contained a hydrophobic core dispersed within a gel matrix (of isotropic interlinked NGs), we investigated whether the composite gels exhibited anisotropic swelling. The anisotropic swelling parameter $(A)$ was calculated using eq 1 (see also cartoon in Figure $3 \mathrm{e}$ and equations in Figure $3 \mathrm{f}$ ). $\mathrm{pH}$ triggered swelling experiments for the nonaligned $\mathrm{DX} \mathrm{NG}_{0.5} /$ $\mathrm{W}_{0.5}$ system confirmed they underwent isotropic swelling (Figure $3 \mathrm{e}(\mathrm{i}, \mathrm{ii}), \alpha_{L}=\alpha_{W}$ and $A=0$ ). Interestingly, the aligned DX $\mathrm{NG}_{0.92} / \mathrm{W}_{0.08}$ (Figure 3e(iii,iv)) and DX NG $\mathrm{NG}_{0.5} / \mathrm{W}_{0.5}$ gels (Figure $3 \mathrm{e}(\mathrm{v}, \mathrm{vi}))$ both exhibited anisotropic swelling. The $A$ values for the latter two systems were $0.11 \pm 0.01$ and $0.31 \pm$ 0.03 , respectively. These $A$ values are shown in Figure $3 f$ (values for $\alpha_{L}, \alpha_{W}$, and $A$ for all of the gels studied in this work are shown in Figure S9). The anisotropic gels swelled least in the direction parallel to worm alignment (lower $\alpha_{L}$ ) because the worm cores were connected and extended within this plane (which contrasts to the perpendicular plane). The hydrophobic worm cores behaved as physical cross-links and opposed swelling. The extent of $\mathrm{DX} \mathrm{NG}_{x} / \mathrm{W}_{1-x}$ anisotropic swelling increased with increasing worm content (i.e., decreasing $x$ ) because the contribution from the physical cross-links of the worm cores increased most in the parallel plane. These results demonstrate the ability to tune anisotropic swelling for these soft aligned gels by varying $x$.

Gel strings containing anisotropic RLPs are attracting considerable interest for biomaterials applications. ${ }^{14}$ As a proof-of-concept study to demonstrate versatility, we used a simple syringe to prepare anisotropic $\mathrm{DX} \mathrm{NG}_{0.50} / \mathrm{W}_{0.5}$ strings. A concentrated $\mathrm{NG}_{0.5} / \mathrm{W}_{0.5}$ physical gel was injected through a syringe needle (inner diameter $=0.84 \mathrm{~mm}$ ). Such extrusion induced worm alignment via shear from the inner syringe needle walls. The physical gel was subsequently converted into the corresponding $\mathrm{DX} \mathrm{NG} \mathrm{NG}_{0.50} / \mathrm{W}_{0.5}$ string by heating at $37^{\circ} \mathrm{C}$. Worm alignment within the $\mathrm{DX} \mathrm{NG}_{0.50} / \mathrm{W}_{0.5}$ composite gel 

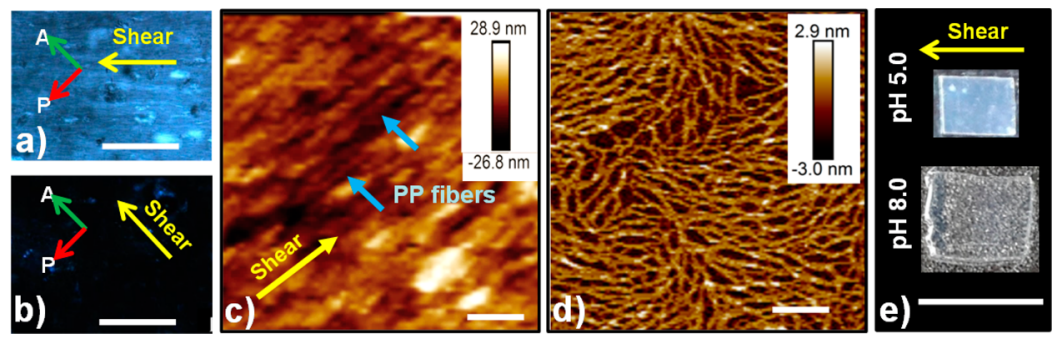

Figure 4. (a, b) POM images for aligned $\mathrm{DX} \mathrm{NG} \mathrm{N}_{0.92} / \mathrm{PP}_{0.08}$ composite gels. (b) The film was dark when the shear direction was parallel to the analyzer. (c, d) AFM images for aligned $\mathrm{DX} \mathrm{NG}_{0.92} / \mathrm{PP}_{0.08}$ and deposited PP fibers, respectively. PP fibers are highlighted in (c). (e) Images for pHdependent swelling of $\mathrm{DX} \mathrm{NG} \mathrm{N}_{0.92} / \mathrm{PP}_{0.08}$. The textured appearance is due to the background. Scale bars: (a, b) $400 \mu \mathrm{m} ;(\mathrm{c}, \mathrm{d}) 200 \mathrm{~nm}$; (e) $6.0 \mathrm{~mm}$.
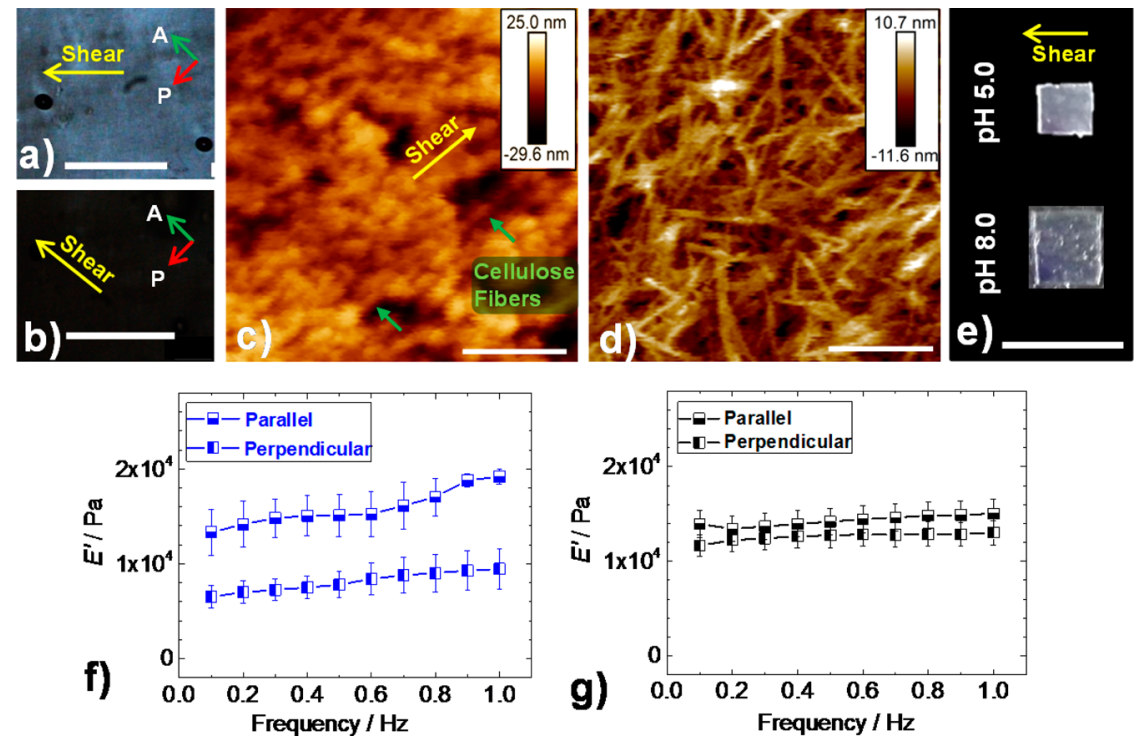

Figure 5. (a, b) POM images for a DX NG $\mathrm{N}_{0.92} / \mathrm{NCC}_{0.08}$ composite gel oriented at different angles with respect to the polarizer and analyzer. (c, d) AFM images for an anisotropic $\mathrm{DX} \mathrm{NG} \mathrm{N}_{0.92} / \mathrm{NCC}_{0.08}$ composite gel and the deposited pristine NCC fibers, respectively. (e) Anisotropic swelling images for a DX NG ${ }_{0.92} / \mathrm{NCC}_{0.08}$ film. Scale bars: (a, b) $400 \mu \mathrm{m}$; (c, d) $200 \mathrm{~nm}$; (e) $6.0 \mathrm{~mm}$. Storage modulus as a function of frequency for (f) anisotropic $\mathrm{DX} \mathrm{NG} 0.92 / \mathrm{NCC}_{0.08}$ film and $(\mathrm{g})$ control NCC-free DX NG $(x=1.0)$ measured parallel or perpendicular to the shear direction used during film formation.

string was confirmed by POM (Figure S10). Formation of an anisotropic composite gel string using such a simple protocol is rare in the literature. ${ }^{14}$

Anisotropic DX NG/RLP Gels Containing Peptides or Nanocrystalline Cellulose. To investigate the effects of intraRLP bond strength and also to establish versatility of our approach, we prepared (linear) shear-aligned DX NGs gel films containing PP fibers. The PPs had a mean diameter of $16 \pm 2$ $\mathrm{nm}$ as determined by AFM. Frequency-sweep rheology data for a pure PP gel dispersion was measured (Figure S11), and $G^{\prime}$ and $G^{\prime \prime}$ did not intersect in the frequency range from 0.1 to 70 $\mathrm{Hz}$. Consequently, we infer that $\tau_{\text {break }}$ was at least $10 \mathrm{~s}$ for this system. This relatively high value is most likely due to the stronger intra-RLP interactions. Figure $4 \mathrm{a}, \mathrm{b}$ shows POM images obtained for $\mathrm{DX} \mathrm{NG}_{0.92} / \mathrm{PP}_{0.08}$. The birefringence evident for this system is similar to that shown in Figure 3a and indicates alignment of the PP fibers. This birefringence disappeared when the sample was rotated through $45^{\circ}$ (Figure 4b). AFM studies were conducted (Figure 4c) and confirmed that the peptide fibers were indeed aligned in the shear direction. In contrast, the AFM image for the parent peptide (Figure 4d) showed interconnected, randomly oriented fibers. The DX $\mathrm{NG}_{0.92} / \mathrm{PP}_{0.08}$ gel films were examined for $\mathrm{pH}$ triggered anisotropic swelling (Figure 4e). The $A$ value of 0.14 \pm 0.02 was significantly greater than the value measured for DX $\mathrm{NG}_{0.92} / \mathrm{W}_{0.08}$. This result indicates that the intra-RLP bond strength dictates the extent of anisotropic swelling.

A RLP system derived from abundant biomass (cellulose) was also examined. The NCCs had a mean diameter and length of $10 \pm 1 \mathrm{~nm}$ and $161 \pm 30 \mathrm{~nm}$, respectively, as determined from AFM. NCC fibers possess an ultrahigh modulus, ${ }^{60}$ have strong covalent and hydrogen bonds within the RLP interior, and exhibit essentially infinite $\tau_{\text {break }}$ values. These RLPs were mixed with NGs for the preparation of anisotropic $\mathrm{DX} \mathrm{NG}_{0.92} /$ $\mathrm{NCC}_{0.08}$ films. Birefringence was observed using POM (Figure $5 \mathrm{a}, \mathrm{b})$, which indicated NCC alignment. AFM data were also obtained for the sheared composite (Figure 5c) and provided evidence for NCC fiber alignment when compared to randomly dispersed NCCs present for a nonaligned sample of deposited pure NCCs (Figure 5d).

Anisotropic $\mathrm{pH}$-dependent swelling (Figure 5e) occurred for the $\mathrm{DX} \mathrm{NG}_{0.92} / \mathrm{NCC}_{0.08}$ composite, and an $A$ value of $0.15 \pm$ 0.02 was measured. Interestingly, this value is indistinguishable from that obtained for $\mathrm{DX} \mathrm{NG} \mathrm{N}_{0.92} \mathrm{PP}_{0.08}$ (Figure $\mathrm{S} 9 \mathrm{~b}$ shows all $A$ values). As mentioned above, anisotropic swelling was caused by restriction of swelling parallel to the direction of shear imposed by intra-RLP bonding and was strongest for the RLPs with high $\tau_{\text {break }}$ values. It appears that once the intra-RLP bond 
strength exceeds a critical value (which corresponds to a $\tau_{\text {break }}$ value greater than $10 \mathrm{~s}$ ) the extent of anisotropic swelling reaches a maximum for a given $x$ value. Following the example of the DX NG $\mathrm{NG}_{x} / \mathrm{W}_{1-x}$ gels (Figure 3f), further increases for $A$ should be possible by increasing $x$. However, a limitation with stiff RLPs is that their dispersion becomes increasingly difficult with increasing concentration because of entanglements. Attempts to prepare $\mathrm{DX} \mathrm{NG}_{x} / \mathrm{W}_{1-x}$ gels with $x$ values of 0.10 or greater were unsuccessful because of NCC entanglements which prevented good dispersion being achieved prior to composite formation. This constraint was not present for the soft worms, and they were able to be loaded into the gels at higher concentrations (e.g., 50\% dry weight), while retaining good dispersion, to give larger anisotropic swelling (Figure 3f). Thus, unexpectedly, the use of soft worm RLPs enabled the preparation of films with the most pronounced anisotropic swelling and highest $A$ value compared to that which could be achieved using hard RLPs.

Dynamic mechanical analysis (DMA) was used to assess the anisotropic mechanical properties of the DX $\mathrm{NG}_{0.92} / \mathrm{NCC}_{0.08}$ composite. For this purpose, we used a DMA equipped with a shear sandwich clamp which applied dynamic planar shear stress. The DMA data (Figure 5f) show that the frequencydependent storage modulus $\left(E^{\prime}\right)$ values for aligned $\mathrm{DX} \mathrm{NG}_{0.92} /$ $\mathrm{NCC}_{0.08}$ measured parallel to the shear direction ranged from $1.3 \times 10^{4}$ to $1.9 \times 10^{4} \mathrm{~Pa}$ and were about a factor of 2 higher than the values of those measured perpendicular to the shear direction (which ranged from $6.5 \times 10^{3}$ to $9.5 \times 10^{3} \mathrm{~Pa}$ ). This implies a doubling of the number of elastically effective chains for the gel in the parallel plane and is due to the contribution of the aligned ultrahigh modulus NCCs. In contrast, the mechanical properties of a control pure DX NG sample $(x=$ 1.0) subject to planar shear did not show any dependence on the direction of applied shear (Figure $5 \mathrm{~g}$ ). The data confirm that these new anisotropic gels also have strongly anisotropic mechanical properties.

Table 1 summarizes the dimensions of the RLPs and NGs used in this study. For all RLP/NG mixtures explored, $q$ was

Table 1. Summary of Structural Parameters for the NGs and RLPs

\begin{tabular}{lccccc} 
system & $\tau_{\text {break }}{ }^{a} / \mathrm{s}$ & diameter $/ \mathrm{nm}$ & length $/ \mathrm{nm}$ & $\xi^{b}$ & $q^{c}$ \\
NG & \multicolumn{7}{c}{} & 40 & & & \\
worm & 1.0 & 35 & 307 & 18.0 & 0.90 \\
PP & $>10$ & 16 & $-{ }^{d}$ & & 0.40 \\
NCC & $\infty$ & 10 & 161 & 9.5 & 0.25
\end{tabular}

${ }^{a}$ Characteristic RLP breaking time. ${ }^{b} \xi=L_{\mathrm{RLP}} / D_{\mathrm{NG}}$, where $L_{\mathrm{RLP}}$ and $D_{\mathrm{RLP}}$ are the length and diameter of the RLPs, respectively. The values shown were obtained from TEM or AFM (see text). $D_{\mathrm{NG}}$ is the diameter of the NGs. The $d_{z}$ value measured at $\mathrm{pH} 7.5$ was used for $D_{\mathrm{NG}} \cdot{ }^{c} q=D_{\mathrm{RLP}} / D_{\mathrm{NG}} \cdot{ }^{d}$ It was not possible to determine the length of the peptide fibers due to their branched nature.

comparable to unity, whereas $\xi$ greatly exceeded unity. It was not possible to determine the precise value of $\xi$ for $\mathrm{NG}_{x} / \mathrm{PP}_{1-x}$ mixtures because the peptides had a pronounced tendency to form branches (Figure 4d). We propose that the use of NGs and RLPs with $q$ reasonably close to unity was a key reason for achieving low shear alignment because of efficient stress transfer from the abundant NGs to the RLPs. Because all three systems exhibited shear-induced alignment, suitable design criteria for future $\mathrm{DX} \mathrm{NG}_{x} / \mathrm{RLP}_{1-x}$ formulations can be suggested as $\xi \geq 9.5$ and $q$ values of approximately 0.2 to 1.0, respectively. Furthermore, the $\tau_{\text {break }}$ values should be at least $1 \mathrm{~s}$. These parameters should enable different RLPs to be utilized, ranging from water-dispersible multiwalled carbon nanotubes ${ }^{75}$ to the tobacco mosaic virus. ${ }^{76}$ Furthermore, NGs are very well-suited for systematic variation in size and/or internal modulus, which should further increase versatility.

An interesting question concerning our new approach for preparing anisotropic hydrogels is whether traditional linear cross-linkable polymer molecules could be used in place of the NGs. Our NGs were designed to efficiently transfer stress to the RLPs due to their $q$ values (which were near to 1 ) and swollen nature. Linear polymer molecules typically have coil diameters that are at least an order of magnitude smaller than the RLP diameters, and hence, the $q$ values would be very large. The latter would contribute to inefficient stress transfer. Linear polymer chains would also deform greatly under shear, dissipating energy, and would transfer stress less efficiently compared to our NGs. In contrast, our internally cross-linked NGs have an inherent elastic component which distributes stress to neighboring RLPs. While aligned RLP composites could be prepared, in principle, using traditional linear crosslinkable polymer chains, it is very likely that much higher shear rates would be needed compared to the low shear rates used in this study with NGs. The ability to use low shear rates is potentially beneficial for future work involving cells.

\section{CONCLUSIONS}

This paper reports a new low-shear approach for the preparation of anisotropic gel composites. Key to the success of our approach was the NGs which enabled low-shear alignment of three types of RLPs with varying intra-RLP stiffness (triblock copolymer worms, PPs, or NCC fibers). The worms were aligned using three different shear regimes: rotational shear, linear shear, and also via extrusion using a syringe. All three types of DX NG/RLP systems exhibited shear-induced birefringence and $\mathrm{pH}$-triggered anisotropic swelling, which demonstrates that our new approach to forming anisotropic composite gels is versatile. The ability of NG particles to lock in the alignment of RLPs at low shear was critical for success, should also apply to other RLPs, and would in principle also be compatible with cells. Remarkably, the softest RLPs (worms) enabled preparation of aligned films with the strongest anisotropic swelling. This unexpected result was due to the ability to disperse the worms within the gel composites at high concentrations. It is highly likely that increases in the extent of anisotropic swelling can be achieved in the future through optimization of the NG and RLP properties. We stress that the NGs were prepared using a scalable, simple emulsion polymerization approach ${ }^{33}$ and that alignment was achieved using inexpensive laboratory equipment. The DX NG/worm gel binary mixtures were not cytotoxic, and anisotropic swelling could be induced at around physiological $\mathrm{pH}$; thus, these formulations are potentially useful new biomaterials. This suggestion also applies to the anisotropic gels containing PPs and NCCs because these RLPs are known to be biocompatible. ${ }^{51,77}$ Possible applications include strengthening and guiding regeneration of load-bearing soft tissues (e.g., intervertebral discs ${ }^{78}$ ). In this context, the observation of worm alignment simply via injection is promising (Figure S10). Furthermore, all of the DX NG/ RLP composite gels exhibited strain-induced birefringence 
when viewed under polarized light, which suggests potential for strain sensor applications. ${ }^{63,79}$

\section{ASSOCIATED CONTENT}

\section{S Supporting Information}

The Supporting Information is available free of charge on the ACS Publications website at DOI: 10.1021/acs.chemmater.7b00110.

Synthesis scheme for NGs and copolymer worms, ${ }^{1} \mathrm{H}$ NMR spectra and GPC for the copolymers and precursors, schematic representation of the shear-aligned gel film preparation method, characterization data for mixtures of NGs and copolymer worms, discussion of assembly and properties of isotropic DX NG/worm gels including SEM and dynamic rheology data, frequencysweep data for concentrated worm dispersion, MTT data for DX NG $\mathrm{NG}_{0.5} / \mathrm{W}_{0.5}$ gel, SAXS data for DX NG $\mathrm{NG}_{0.5} / \mathrm{W}_{0.5}$ gels, swelling ratios and $A$ values for all gels studied, photographs of $\mathrm{DX} \mathrm{NG}_{0.5} / \mathrm{W}_{0.5}$ string after injection through a syringe needle, frequency-sweep rheology data for a peptide gel (PDF)

\section{AUTHOR INFORMATION}

\section{Corresponding Authors}

*E-mail: amirhossein.milani@manchester.ac.uk (A.H.M.).

*E-mail: brian.saunders@manchester.ac.uk (B.R.S.).

\section{ORCID $\odot$}

Brian R. Saunders: 0000-0003-1410-2967

Steven P. Armes: 0000-0002-8289-6351

Notes

The authors declare no competing financial interest.

\section{ACKNOWLEDGMENTS}

B.R.S. and S.P.A. would like to thank the EPSRC for funding this research (EP/K030949/1 and EP/K03071X/1). O.O.M. thanks Anton Paar Ltd. (Graz, Austria) for the donated rheometer.

\section{REFERENCES}

(1) Gong, J. P.; Katsuyama, Y.; Kurokawa, T.; Osada, Y. DoubleNetwork Hydrogels with Extremely High Mechanical Strength. Adv. Mater. 2003, 15, 1155-1158.

(2) Haque, M. A.; Kurokawa, T.; Gong, J. P. Super tough double network hydrogels and their application as biomaterials. Polymer 2012, $53,1805-1822$.

(3) Haraguchi, K.; Takehisa, T. Nanocomposite Hydrogels: A Unique Organic-Inorganic Network Structure with Extraordinary Mechanical, Optical, and Swelling/De-swelling Properties. Adv. Mater. 2002, 14, 1120-1124.

(4) Sun, J.-Y.; Zhao, X.; Illeperuma, W. R. K.; Chaudhuri, O.; Oh, K. H.; Mooney, D. J.; Vlassak, J. J.; Suo, Z. Highly stretchable and tough hydrogels. Nature 2012, 489, 133-136.

(5) Yasuda, K.; Ping Gong, J.; Katsuyama, Y.; Nakayama, A.; Tanabe, Y.; Kondo, E.; Ueno, M.; Osada, Y. Biomechanical properties of hightoughness double network hydrogels. Biomaterials 2005, 26, 44684475.

(6) Jeon, I.; Cui, J.; Illeperuma, W. R. K.; Aizenberg, J.; Vlassak, J. J. Extremely Stretchable and Fast Self-Healing Hydrogels. Adv. Mater. 2016, 28, 4678-4683.

(7) Taylor, D. L.; in het Panhuis, M. Self-Healing Hydrogels. Adv. Mater. 2016, 28, 9060-9093.

(8) Rodell, C. B.; Dusaj, N. N.; Highley, C. B.; Burdick, J. A. Injectable and Cytocompatible Tough Double-Network Hydrogels through Tandem Supramolecular and Covalent Crosslinking. Adv. Mater. 2016, 28, 8419-8424.

(9) Luo, F.; Sun, T. L.; Nakajima, T.; King, D. R.; Kurokawa, T.; Zhao, Y.; Ihsan, A. B.; Li, X.; Guo, H.; Gong, J. P. Strong and Tough Polyion-Complex Hydrogels from Oppositely Charged Polyelectrolytes: A Comparative Study with Polyampholyte Hydrogels. Macromolecules 2016, 49, 2750-2760.

(10) Feldner, T.; Häring, M.; Saha, S.; Esquena, J.; Banerjee, R.; Díaz, D. D. Supramolecular Metallogel That Imparts Self-Healing Properties to Other Gel Networks. Chem. Mater. 2016, 28, 3210-3217.

(11) Moutos, F. T.; Freed, L. E.; Guilak, F. A biomimetic threedimensional woven composite scaffold for functional tissue engineering of cartilage. Nat. Mater. 2007, 6, 162-167.

(12) Wingfield, C.; Baski, A.; Bertino, M. F.; Leventis, N.; Mohite, D. P.; Lu, H. Fabrication of Sol-Gel Materials with Anisotropic Physical Properties by Photo-Cross-Linking. Chem. Mater. 2009, 21, 21082114.

(13) Yue, Y.; Kurokawa, T.; Haque, M. A.; Nakajima, T.; Nonoyama, T.; Li, X.; Kajiwara, I.; Gong, J. P. Mechano-actuated ultrafast fullcolour switching in layered photonic hydrogels. Nat. Commun. 2014, 5, 4659.

(14) Zhang, S.; Greenfield, M. A.; Mata, A.; Palmer, L. C.; Bitton, R.; Mantei, J. R.; Aparicio, C.; de la Cruz, M. O.; Stupp, S. I. A selfassembly pathway to aligned monodomain gels. Nat. Mater. 2010, 9, 594-601.

(15) Merzlyak, A.; Indrakanti, S.; Lee, S.-W. Genetically Engineered Nanofiber-Like Viruses For Tissue Regenerating Materials. Nano Lett. 2009, 9, 846-852.

(16) Takashima, Y.; Hatanaka, S.; Otsubo, M.; Nakahata, M.; Kakuta, T.; Hashidzume, A.; Yamaguchi, H.; Harada, A. Expansioncontraction of photoresponsive artificial muscle regulated by hostguest interactions. Nat. Commun. 2012, 3, 1270.

(17) Pei, X.; Zan, T.; Li, H.; Chen, Y.; Shi, L.; Zhang, Z. Pure Anisotropic Hydrogel with an Inherent Chiral Internal Structure Based on the Chiral Nematic Liquid Crystal Phase of Rodlike Viruses. ACS Macro Lett. 2015, 4, 1215-1219.

(18) Wu, Z. L.; Kurokawa, T.; Sawada, D.; Hu, J.; Furukawa, H.; Gong, J. P. Anisotropic Hydrogel from Complexation-Driven Reorientation of Semirigid Polyanion at $\mathrm{Ca} 2+$ Diffusion Flux Front. Macromolecules 2011, 44, 3535-3541.

(19) Chung, C.-Y.; Bien, H.; Entcheva, E. The Role of Cardiac Tissue Alignment in Modulating Electrical Function. J. Cardio. Electrophys. 2007, 18, 1323-1329.

(20) Byun, M.; Santangelo, C. D.; Hayward, R. C. Swelling-driven rolling and anisotropic expansion of striped gel sheets. Soft Matter 2013, 9, 8264-8273.

(21) Huang, Z.; Lee, H.; Lee, E.; Kang, S.-K.; Nam, J.-M.; Lee, M. Responsive nematic gels from the self-assembly of aqueous nanofibres. Nat. Commun. 2011, 2, 459.

(22) Shigekura, Y.; Chen, Y. M.; Furukawa, H.; Kaneko, T.; Kaneko, D.; Osada, Y.; Gong, J. P. Anisotropic Polyion-Complex Gels from Template Polymerization. Adv. Mater. 2005, 17, 2695-2699.

(23) Davies, S. J. A.; Goucher, D. R.; Doller, C.; Silver, J. Robust Regeneration of Adult Sensory Axons in Degenerating White Matter of the Adult Rat Spinal Cord. J. Neurosci. 1999, 19, 5810-5822.

(24) Draper, E. R.; Mykhaylyk, O. O.; Adams, D. J. Aligning selfassembled gelators by drying under shear. Chem. Commun. 2016, 52, 6934-6937.

(25) Haque, M. A.; Kamita, G.; Kurokawa, T.; Tsujii, K.; Gong, J. P. Unidirectional Alignment of Lamellar Bilayer in Hydrogel: OneDimensional Swelling, Anisotropic Modulus, and Stress/Strain Tunable Structural Color. Adv. Mater. 2010, 22, 5110-5114.

(26) Sehaqui, H.; Ezekiel Mushi, N.; Morimune, S.; Salajkova, M.; Nishino, T.; Berglund, L. A. Cellulose Nanofiber Orientation in Nanopaper and Nanocomposites by Cold Drawing. ACS Appl. Mater. Interfaces 2012, 4, 1043-1049.

(27) Wang, P.-X.; Hamad, W. Y.; MacLachlan, M. J. Structure and transformation of tactoids in cellulose nanocrystal suspensions. Nat. Commun. 2016, 7, 11515. 
(28) Kong, H. J.; Smith, M. K.; Mooney, D. J. Designing alginate hydrogels to maintain viability of immobilized cells. Biomaterials 2003, 24, 4023-4029.

(29) Chau, M.; De France, K. J.; Kopera, B.; Machado, V. R.; Rosenfeldt, S.; Reyes, L.; Chan, K. J. W.; Förster, S.; Cranston, E. D.; Hoare, T.; Kumacheva, E. Composite Hydrogels with Tunable Anisotropic Morphologies and Mechanical Properties. Chem. Mater. 2016, 28, 3406-3415.

(30) Inomata, K.; Iguchi, Y.; Mizutani, K.; Sugimoto, H.; Nakanishi, E. Anisotropic Swelling Behavior Induced by Helix-Coil Transition in Liquid Crystalline Polypeptide Gels. ACS Macro Lett. 2012, 1, 807810.

(31) van Kuringen, H. P. C.; Leijten, Z. J. W. A.; Gelebart, A. H.; Mulder, D. J.; Portale, G.; Broer, D. J.; Schenning, A. P. H. J. Photoresponsive Nanoporous Smectic Liquid Crystalline Polymer Networks: Changing the Number of Binding Sites and Pore Dimensions in Polymer Adsorbents by Light. Macromolecules 2015, 48, 4073-4080.

(32) Kempe, M. D.; Scruggs, N. R.; Verduzco, R.; Lal, J.; Kornfield, J. A. Self-assembled liquid-crystalline gels designed from the bottom up. Nat. Mater. 2004, 3, 177-182.

(33) Milani, A. H.; Saunders, J. M.; Nguyen, N. T.; Ratcliffe, L. P. D.; Adlam, D. J.; Freemont, A. J.; Hoyland, J. A.; Armes, S. P.; Saunders, B. R. Synthesis of polyacid nanogels: $\mathrm{pH}$-responsive sub-100 $\mathrm{nm}$ particles for functionalisation and fluorescent hydrogel assembly. Soft Matter 2017, 13, 1554.

(34) Tan, B. H.; Pelton, R. H.; Tam, K. C. Microstructure and rheological properties of thermo-responsive poly $(\mathrm{N}$-isopropylacrylamide) microgels. Polymer 2010, 51, 3238-3243.

(35) Richtering, W. Responsive Emulsions Stabilized by StimuliSensitive Microgels: Emulsions with Special Non-Pickering Properties. Langmuir 2012, 28, 17218-17229.

(36) Hendrickson, G. R.; Lyon, L. A. Microgel Translocation through Pores under Confinement. Angew. Chem., Int. Ed. 2010, 49, 21932197.

(37) Saunders, B. R.; Laajam, N.; Daly, E.; Teow, S.; Hu, X.; Stepto, R. Microgels: From responsive polymer colloids to biomaterials. Adv. Colloid Interface Sci. 2009, 147-148, 251-262.

(38) Wu, H.-Q.; Wang, C.-C. Biodegradable Smart Nanogels: A New Platform for Targeting Drug Delivery and Biomedical Diagnostics. Langmuir 2016, 32, 6211-6225.

(39) Guu, D.; Dhont, J. K. G.; Lettinga, M. P. Dispersions and mixtures of particles with complex architectures in shear flow. Eur. Phys. J.: Spec. Top. 2013, 222, 2739-2755.

(40) Croce, V.; Cosgrove, T.; Dreiss, C. A.; King, S.; Maitland, G.; Hughes, T. Giant Micellar Worms under Shear: A Rheological Study Using SANS. Langmuir 2005, 21, 6762-6768.

(41) Frounfelker, B. D.; Kalur, G. C.; Cipriano, B. H.; Danino, D.; Raghavan, S. R. Persistence of Birefringence in Sheared Solutions of Wormlike Micelles. Langmuir 2009, 25, 167-172.

(42) Winkler, R. G.; Mussawisade, K.; Ripoll, M.; Gompper, G. Rodlike colloids and polymers in shear flow: a multi-particle-collision dynamics study. J. Phys.: Condens. Matter 2004, 16, S3941-S3954.

(43) Sugihara, S.; Blanazs, A.; Armes, S. P.; Ryan, A. J.; Lewis, A. L. Aqueous Dispersion Polymerization: A New Paradigm for in Situ Block Copolymer Self-Assembly in Concentrated Solution. J. Am. Chem. Soc. 2011, 133, 15707-15713.

(44) Fielding, L. A.; Lane, J. A.; Derry, M. J.; Mykhaylyk, O. O.; Armes, S. P. Thermo-responsive Diblock Copolymer Worm Gels in Non-polar Solvents. J. Am. Chem. Soc. 2014, 136, 5790-5798.

(45) Warren, N. J.; Armes, S. P. Polymerization-Induced SelfAssembly of Block Copolymer Nano-objects via RAFT Aqueous Dispersion Polymerization. J. Am. Chem. Soc. 2014, 136, 1017410185.

(46) Verber, R.; Blanazs, A.; Armes, S. P. Rheological studies of thermo-responsive diblock copolymer worm gels. Soft Matter 2012, 8, 9915-9922.

(47) Jones, E. R.; Semsarilar, M.; Blanazs, A.; Armes, S. P. Efficient Synthesis of Amine-Functional Diblock Copolymer Nanoparticles via
RAFT Dispersion Polymerization of Benzyl Methacrylate in Alcoholic Media. Macromolecules 2012, 45, 5091-5098.

(48) Canning, S. L.; Smith, G. N.; Armes, S. P. A Critical Appraisal of RAFT-Mediated Polymerization-Induced Self-Assembly. Macromolecules 2016, 49, 1985-2001.

(49) Charleux, B.; Delaittre, G.; Rieger, J.; D’Agosto, F. Polymerization-Induced Self-Assembly: From Soluble Macromolecules to Block Copolymer Nano-Objects in One Step. Macromolecules 2012, 45, 6753-6765.

(50) Mable, C. J.; Thompson, K. L.; Derry, M. J.; Mykhaylyk, O. O.; Binks, B. P.; Armes, S. P. ABC Triblock Copolymer Worms: Synthesis, Characterization, and Evaluation as Pickering Emulsifiers for Millimeter-Sized Droplets. Macromolecules 2016, 49, 7897-7907.

(51) Jayawarna, V.; Ali, M.; Jowitt, T. A.; Miller, A. F.; Saiani, A.; Gough, J. E.; Ulijn, R. V. Nanostructured Hydrogels for ThreeDimensional Cell Culture Through Self-Assembly of Fluorenylmethoxycarbonyl-Dipeptides. Adv. Mater. 2006, 18, 611-614.

(52) Banwell, E. F.; Abelardo, E. S.; Adams, D. J.; Birchall, M. A.; Corrigan, A.; Donald, A. M.; Kirkland, M.; Serpell, L. C.; Butler, M. F.; Woolfson, D. N. Rational design and application of responsive [alpha]helical peptide hydrogels. Nat. Mater. 2009, 8, 596-600.

(53) Shirbin, S. J.; Karimi, F.; Chan, N. J.-A.; Heath, D. E.; Qiao, G. G. Macroporous Hydrogels Composed Entirely of Synthetic Polypeptides: Biocompatible and Enzyme Biodegradable 3D Cellular Scaffolds. Biomacromolecules 2016, 17, 2981-2991.

(54) Clarke, K. C.; Lyon, L. A. Microgel Surface Modification with Self-Assembling Peptides. Macromolecules 2016, 49, 5366-5373.

(55) King, P. J. S.; Lizio, M. G.; Booth, A.; Collins, R. F.; Gough, J. E.; Miller, A. F.; Webb, S. J. A modular self-assembly approach to functionalised beta-sheet peptide hydrogel biomaterials. Soft Matter 2016, 12, 1915-1923.

(56) Bowerman, C. J.; Nilsson, B. L. Review self-assembly of amphipathic $\beta$-sheet peptides: Insights and applications. Biopolymers 2012, 98, 169-184.

(57) Thérien-Aubin, H.; Wang, Y.; Nothdurft, K.; Prince, E.; Cho, S.; Kumacheva, E. Temperature-Responsive Nanofibrillar Hydrogels for Cell Encapsulation. Biomacromolecules 2016, 17, 3244-3251.

(58) Gregorczyk, K. E.; Pickup, D. F.; Sanz, M. G.; Irakulis, I. A.; Rogero, C.; Knez, M. Tuning the Tensile Strength of Cellulose through Vapor-Phase Metalation. Chem. Mater. 2015, 27, 181-188.

(59) Eichhorn, S. J. Cellulose nanowhiskers: promising materials for advanced applications. Soft Matter 2011, 7, 303-315.

(60) Azizi Samir, M. A. S.; Alloin, F.; Dufresne, A. Review of Recent Research into Cellulosic Whiskers, Their Properties and Their Application in Nanocomposite Field. Biomacromolecules 2005, 6, 612-626.

(61) Yang, J.; Han, C.-R.; Duan, J.-F.; Xu, F.; Sun, R.-C. Mechanical and Viscoelastic Properties of Cellulose Nanocrystals Reinforced Poly(ethylene glycol) Nanocomposite Hydrogels. ACS Appl. Mater. Interfaces 2013, 5, 3199-3207.

(62) Parker, R. M.; Frka-Petesic, B.; Guidetti, G.; Kamita, G.; Consani, G.; Abell, C.; Vignolini, S. Hierarchical Self-Assembly of Cellulose Nanocrystals in a Confined Geometry. ACS Nano 2016, 10, 8443-8449.

(63) Maeda, A.; Inoue, T.; Yamaguchi, M. Dynamical rigidity of cellulose derivatives in melts. Polym. J. (Tokyo, Jpn.) 2014, 46, 149154.

(64) Pellens, L.; Vermant, J.; Mewis, J. Deviations from the StressOptical Rule in Telechelic Associative Polymer Solutions. Macromolecules 2005, 38, 1911-1918.

(65) Bondeson, D.; Mathew, A.; Oksman, K. Optimization of the isolation of nanocrystals from microcrystalline cellulose by acid hydrolysis. Cellulose 2006, 13, 171-180.

(66) Lovett, J. R.; Ratcliffe, L. P. D.; Warren, N. J.; Armes, S. P.; Smallridge, M. J.; Cracknell, R. B.; Saunders, B. R. A Robust CrossLinking Strategy for Block Copolymer Worms Prepared via Polymerization-Induced Self-Assembly. Macromolecules 2016, 49, 2928-2941.

(67) Ilavsky, J. Nika: software for two-dimensional data reduction. J. Appl. Crystallogr. 2012, 45, 324-328. 
(68) Mykhaylyk, O. O.; Warren, N. J.; Parnell, A. J.; Pfeifer, G.; Laeuger, J. Applications of shear-induced polarized light imaging (SIPLI) technique for mechano-optical rheology of polymers and soft matter materials. J. Polym. Sci., Part B: Polym. Phys. 2016, 54, 21512170.

(69) Wang, C.; Hu, Z.; Chen, Y.; Li, Y. Swelling Behavior of Polymer Gels with Built-In Anisotropy near the Volume-Phase Transition Point. Macromolecules 1999, 32, 1822-1827.

(70) Mykhaylyk, O. O.; Warren, N. J.; Parnell, A. J.; Pfeifer, G.; Laeuger, J. Applications of shear-induced polarized light imaging (SIPLI) technique for mechano-optical rheology of polymers and soft matter materials. J. Polym. Sci., Part B: Polym. Phys. 2016, 54, 21512170.

(71) Mykhaylyk, O. O. Time-resolved polarized light imaging of sheared materials: application to polymer crystallization. Soft Matter 2010, 6, 4430-4440.

(72) Mykhaylyk, O. O.; Parnell, A. J.; Pryke, A.; Fairclough, J. P. A. Direct Imaging of the Orientational Dynamics of Block Copolymer Lamellar Phase Subjected to Shear Flow. Macromolecules 2012, 45, 5260-5272.

(73) Haque, M. A.; Gong, J. P. Multi-functions of hydrogel with bilayer-based lamellar structure. React. Funct. Polym. 2013, 73, 929935.

(74) Roe, R. J. Methods of X-ray and Neutron Scattering in Polymer Science; Oxford University Press: New York, 2000.

(75) Kennedy, A. J.; Hull, M. S.; Steevens, J. A.; Dontsova, K. M.; Chappell, M. A.; Gunter, J. C.; Weiss, C. A. Factors influencing the partitioning and toxicity of nanotubes in the aquatic environment. Environ. Toxicol. Chem. 2008, 27, 1932-1941.

(76) Maier, E. E.; Krause, R.; Deggelmann, M.; Hagenbuechle, M.; Weber, R.; Fraden, S. Liquidlike order of charged rodlike particle solutions. Macromolecules 1992, 25, 1125-1133.

(77) Zoppe, J. O.; Peresin, M. S.; Habibi, Y.; Venditti, R. A.; Rojas, O. J. Reinforcing Poly ( $\varepsilon$-caprolactone) Nanofibers with Cellulose Nanocrystals. ACS Appl. Mater. Interfaces 2009, 1, 1996-2004.

(78) Milani, A. H.; Freemont, A. J.; Hoyland, J. A.; Adlam, D. J.; Saunders, B. R. Injectable Doubly Cross-Linked Microgels for Improving the Mechanical Properties of Degenerated Intervertebral Discs. Biomacromolecules 2012, 13, 2793-2801.

(79) Shikinaka, K.; Koizumi, Y.; Shigehara, K. Mechanical/optical behaviors of imogolite hydrogels depending on their compositions and oriented structures. J. Appl. Polym. Sci. 2014, 132, 41691. 